\title{
Emergency EC-IC bypass for symptomatic atherosclerotic ischemic stroke
}

Tetsuyoshi Horiuchi, Junpei Nitta, Shigetoshi Ishizaka,

Kohei Kanaya, Takao Yanagawa, and Kazuhiro Hongo.

Department of Neurosurgery,

Shinshu University School of Medicine,

Matsumoto, Japan

\section{Correspondence to:}

Tetsuyoshi Horiuchi, M.D.

Department of Neurosurgery,

Shinshu University School of Medicine,

3-1-1 Asahi, Matsumoto 390-8621, Japan

Telephone: +81-263-37-2690, Fax: +81-263-37-0480

E-mail: tetuyosi@shinshu-u.ac.jp 


\begin{abstract}
Previous studies have shown that extracranial-intracranial (EC-IC) bypass surgery has no preventive effect on subsequent ipsilateral ischemic stroke in patients with symptomatic atherosclerotic internal carotid occlusion and hemodynamic cerebral ischemia. A few studies have assessed whether an urgent EC-IC bypass surgery is an effective treatment for main trunk stenosis or occlusion in acute stage. The authors retrospectively reviewed 58 consecutive patients who underwent urgent EC-IC bypass for symptomatic internal carotid artery or the middle cerebral artery stenosis or occlusion between January 2003 and December 2011. Clinical characteristics and neuroimagings were evaluated and analyzed. Based on preoperative angiogram, responsible lesions were the internal carotid artery in $19(32.8 \%)$ patients and the middle cerebral artery in $39(67.2 \%)$. No hemorrhagic complication occurred. Sixty nine percent of patients showed improvement of neurological function after surgery and $74.1 \%$ of patients had favorable outcome. Unfavorable outcome was associated with insufficient collateral flow and new infarction after bypass surgery.
\end{abstract}

Key words: anastomosis, athero-occlusive disease, extracranial-intracranial bypass, revascularization 


\section{Introduction}

Recent randomized clinical trial in patients with atherosclerotic internal carotid artery (ICA) occlusion to medical or extracranial-intracranial (EC-IC) bypass surgery in chronic stage found that superficial temporal artery (STA)- middle cerebral artery (MCA) bypass provided no benefit over medical therapy [8]. By contrast, surgical revascularization may be effective to some patients who suffered from medication-resistance hemodynamic stroke in acute stage $[3-7,10]$. However, safety and efficacy of surgical revascularization for ischemic stroke in acute stage is still controversial. We have reported the efficacy of urgent embolectomy for the ICA or MCA occlusion caused by embolism [4,6]. In the present study, we analyzed our experience with early EC-IC bypass in acute ischemic stroke and stroke in progress due to arteriosclerosis.

\section{Patients and Methods}

Patient population: From January 2003 to December 2011, 101 patients with steno-occlusive disease at the anterior circulation underwent EC-IC bypass. Of the 101 patients who underwent 104 procedures, there were 59 patients treated with urgent EC-IC bypass to treat acute ischemic stroke. Crescendo transient ischemic attack resistance to medical treatment and progressive ischemic stroke due to the steno-occlusive disease of ICA or MCA were also treated with direct bypass. The EC-IC bypass for moyamoya or atherosclerotic patients in the chronic phase were excluded. We retrospectively analyzed hospital records, neuroimaging studies, and operative reports. The study protocol was approved by the institutional ethics committee. 
Management of patients: On admission, the patient presenting with speech disturbance, motor weakness, and/or cognitive dysfunction initially underwent computed tomography (CT). Based on neurological findings and CT, main trunk stenosis or occlusion was suspected. Main artery stenosis or occlusion at the ICA or MCA was generally detected using magnetic resonance imaging (MRI) and confirmed with cerebral angiography. Embolus or atherosclerosis was diagnosed based on the past history such as atrial fibrillation and neuroimages. The embolic stroke in patients with insufficient endovascualr recanalization was treated by the open embolectomy $[3,4,6,8]$. The EC-IC bypass was performed for steno-occlusive athelosclerosis. When it was difficult to distinguish the atherosclerosis from the embolus preoperatively, the affected artery was directly visualized and the appropriate surgery was carried out. Surgical indications of urgent revascularization were previously reported in detail $[4,6]$. Briefly, inclusion criteria were: (1) the patient who needed no supports for daily life before ictus, (2) severe neurological deficits with/without cognitive dysfunction were present, (3) diffusion weighted images revealed no abnormal findings or minimum early signs of ischemia on admission, (4) main artery stenosis or occlusion causing symptoms was confirmed, (5) medical treatment (bolus intravenous injection of heparin (5000 IU) followed by weight-based dose of heparin infusion and oral antiplatelets drugs such as aspirin and clopidogrel) failed sufficient recanalization and progression of ischemic symptoms, and (6) follow-up neuroimagings showed no new infarction at the affected vascular territory.

Clinical characteristics and outcomes: The location of stenosis or occlusion was divided into 5 groups: cervical ICA, intradural ICA, proximal M1 segment of MCA, distal M1 segment, and M2 segment. Preoperative neurological conditions of patients 
were assessed by Glasgow Coma Scale. Patients' outcome at discharge was evaluated with Glasgow Outcome Scale and divided in two categories: favorable outcome (good recovery and moderate disability) or unfavorable outcome (severe disability, vegetative state, and dead). Collateral flow through anterior communicating artery, posterior communicating artery, and/or leptomeningeal anastomosis were checked by angiography and categorized into three groups: (1) good flow: there was no flow delay comparing with non-affected side in the arterial phase; (2) moderate flow: some arterial flow delay in the arterial phase without avascualr area in the capillary phase; (3) poor flow: presence of avasuclar area in the capillary phase. Postoperatively, infarction area was evaluated and followed using CT and/or MRI.

Surgical techniques: EC-IC bypass was undertaken to restore cerebral blood flow at the affected territory. The great majority of patients underwent urgent single or double STA-MCA anastomosis. Generally, M4 segment was selected for the recipient. Based on intraoperative qualitative indocyanine green (ICG) videoangiography and/or Doppler flowmeter findings, single or double anastomois was carried out. Control ICG videoangiography before anastomosis confirmed recipient cortical arteries which had slow antegrade or retrograde filling due to the atherosclerosis. The single bypass was performed between the STA and one of the recipients. After the first bypass was complete, follow-up ICG determined if the blood flow had improved. If the blood flow increase was observed at both frontal and temporal cortical arteries, we judged that the single bypass was sufficient. By contrast, when the blood flow increased at only frontal (or temporal) lobe, the second bypass was carried out between the STA and the one of cortical arteries at the temporal (or frontal) lobe. 
Statistical Analysis: Univariate analyses employed the Pearson's $\chi^{2}$ test (or Fisher's exact test when the expected frequency of events was less than 5) and unpaired t test to evaluate statistical significance between favorable and unfavorable outcomes of patients. Data analysis was carried out using a statistical software package (PASW Statistics 18, SPSS Japan). Significance was assigned at $P<0.05$.

\section{Results}

Direct bypass was not performed in one patient because the patient did not have sufficient recipient vessels on the exposed brain surface. Therefore, 58 patients were enrolled on this urgent EC-IC bypass study. Although intravenous tissue plasminogen activator (tPA) was used in patients with embolic stroke, ischemic stroke due to atherosclerosis was not treated using tPA. Therefore, all patients in the present study did not undergo intravenous tPA therapy. Among 58 patients, 34 patients $(58.6 \%)$ underwent bypass surgery as soon as possible on Day 0 . The majority of other 24 patients underwent the surgical treatment up to Day 2. Fifteen (25.9\%) and 9 patients $(15.5 \%)$ presented with progressive ischemic stroke and crescendo transient ischemic attack, respectively. There were 40 male and 18 female patients, whose ages ranged from 35 to 87 years old $(70.5 \pm 10.5$ years; mean \pm SD). Table 1 shows demographic data of the patients. Forty three $(74.1 \%)$ of 58 patients presented with hemiparesis, 38 $(65.5 \%)$ with consciousness disturbance, and 20 (34.5\%) with aphasia. In 3 cases, open embolectomy was initiated for thrombo-embolic lesions. However, recanalization was not present and bypass procedure was carried out. Single bypass was achieved in 37 patients and double bypasses in 21. The posterior auricular artery [2] was used as the donor artery in only one patient because the sufficient STA was not present. The responsible arteries were ICA in 19 patients and MCA in 39. Neurological functions 
improved in $40(69.0 \%)$ patients after surgery. The bypass surgery did not prevent the new infarction at the affected vascular territory in 23 patients $(39.7 \%)$. Complications during perioperative periods are presented in Table 2. Major complications happened in 4 patients. They included the perforator infarction (Case 2) probably due to hemodynamic change ${ }^{1}$ and remote infarction (posterior cerebral artery territory). Two patients developed myocardial infarction and one died due to the myocardial infarction. No hemorrhagic infarction happened after bypass. Postoperative MR angiography and/or angiogram was performed on 38 of the 58 patients, 37 of whom had patent bypasses.

Forty three (74.1\%) and 15 (25.9\%) patients had favorable (good recovery: 29, moderate disability: 14) and unfavorable (severe disability: 14 , vegetative state: 0 , dead: 1) outcomes, respectively. Favorable and unfavorable outcomes after urgent STA-MCA bypass were detailed in Table 3. Univariate analysis identified 2 significant associations for unfavorable outcome: insufficient collateral flow $(\mathrm{P}=.003)$ and new infarction after surgery $(\mathrm{P}<.001)$. However, age, sex, side, affected artery, and single bypass were not risk factors of unfavorable outcome.

\section{Illustrative case 1}

This 70-year-old man presented with right hemiparesis, aphasia, agnosia, and acaluculia. MRI demonstrated small ischemic lesions on diffusion-weighted images (Figure 1) and subsequent angiograms disclosed the left M1proximal occlusion with moderate leptomenigeal collateral flow (Figure 1). Medical treatment was not effective and surgical treatment was performed on Day 0. He underwent STA-MCA bypass. After fronto-temporal craniotomy, retrograde blood flow of cortical arteries was confirmed. Firstly, the parietal branch was anastomosed to the precentral artery with 18 minutes 
occlusion time. After anastomosis, blood flow increased in ascending frontal and temporal cortical arteries, but not parietal arteries. Therefore, second bypass was carried out between the frontal branch and the anterior parietal artery. The second occlusion time of M4 arteries was 22 minutes. Postoperatively, he recovered well and neurological symptoms were resolved on Day 1. No new infarction was seen on CT and MRI. He was discharged without neurological deficits on Day 9. Postoperative angiogram 3 months later revealed the STA fed the left MCA territory (Figure 2).

\section{Illustrative case 2}

A 70-year-old woman was transferred with global aphasia and without motor weakness. Neuroimages disclosed an M2 severe stenosis (Figure 3). The lateral lenticulostriate arteries originated from the stenotic portion (Figure 3). She underwent single STA-MCA anastomosis uneventfully. After surgery, aphasia improved; however, right hemiparesis developed. Follow-up CT scans revealed new infarction in the basal ganglia and coronal radiation fed by the lateral lenticulostriate arteries (Figure 4). Postoperative angiogram was not performed. She was transferred to a rehabilitation hospital.

\section{Discussion}

Since there are only a few reports concerning urgent EC-IC bypass in atherosclerotic steno-occlusive disease, we retrospectively evaluated 58 consecutive patients who underwent urgent EC-IC bypass surgery in an attempt to augment blood flow of ischemic brain.

In general, early antegrade recanalization is the optimal treatment for ischemic stroke. Although intravenous, intraarterial, and mechanical thrombolysis are accepted as a standard treatment in acute phase, they are often unsatisfactory. Additionally, late 
antegrade recanalization has a risk of reperfusion-related hemorrhagic complication. We previously reported the efficacy of open embolectomy in patients with cardioembolic MCA or ICA occlusion [4,6]. In patients with symptomatic ICA or MCA arteriosclerosis except for segmental cervical ICA stenosis, antegrade surgical revascularization is usually difficult. Therefore, EC-IC bypass is applied and augments cerebral blood flow $(\mathrm{CBF})$ with low-flow reperfusion. Emergency EC-IC bypass would improve neurological deficits resulting from a small amount of blood flow into ischemic brain related to steno-occlusion of arteriosclerosis. Nussbaum et al. [7] demonstrated excellent results of emergency bypass in 13 carefully selected patients. Majority of patients $(84.6 \%)$ suffered ICA or MCA dissection, not atherosclerosis. All patients improved from their preoperative neurological status and $8(61.5 \%)$ patients recovered completely within 6 months after surgery. New infarction at the affected side was observed in only one patient. Hwang and collegues [5] presented their urgent EC-IC bypass results in 9 patients with relatively small infarction $(<30 \mathrm{~mL})$, increased perfusion/diffusion mismatch (> 120\%), and fluctuating or progressive symptoms resistance to medical or endovascular therapy. They concluded that urgent bypass was useful in highly selected patients evaluated with multimodal and advanced CT and MRI. The CBF study is one of useful tools to assess necessity of revascularization surgery. Recent advances of MRI perfusion images without contrast medium demonstrated the potential of becoming the standard tool to estimate regional CBF in acute ischemic stroke [9]. However, it is very difficult to judge a treatable penumbra area.

In the present study, we found that $69.0 \%$ of patients showed an improvement of neurological function after surgery although new infarction area on CT or MRI developed in $40 \%$ patients after bypass surgery. The risk factor of unfavorable outcome 
was new infarction and collateral flow. However, age, side, sex, and affected location were not associated with unfavorable outcome. The importance of collateral flow has been reported in patients undergoing urgent cerebral revascularization surgery $[3,4,11]$. In this study, collateral flow was evaluated with cerebral angiography and therapeutic penumbra was assumed according to follow-up CT scan.

There were no hemorrhagic infarctions as a complication in this study. This result was consistent with recent investigations [5,7]. However, previous study [11] demonstrated that 4 (11\%) of 35 patients had hemorrhagic infarction after urgent bypass surgery. This study probably included patients suffering from cardio-embolic stroke. In general, atherosclerosis gradually affects main trunk arteries compared with embolic stroke. We reported the hemorrhagic infarction after open embolectomy happened in 3 (10\%) of 30 patients [3]. Therefore, low flow bypass surgery comparing with embolectomy and carotid endarterectomy may be a relative low risk of hemorrhagic transformation. According to recent publications [5,7] of urgent EC-IC bypass, no reperfusion-related complications were observed. In the present study, perforator infarction occurred after bypass surgery in the presented Case 2. Severe stenosis may result in occlusion due to the counter blood supply through the anastomosis. Our findings indicated that apart from improvement of cortical blood flow, perforating artery occlusion can also occur after bypass surgery. Therefore, special attention should be paid in patients with perforating arteries originating from the severely stenotic artery.

\section{Conclusions}

We reviewed 58 patients who underwent urgent EC-IC bypass and presented the surgical results. This study is the largest series to date. However, the present study 
EC-IC bypass

has a number of shortcomings that should be addressed. It does not include sufficient CBF evaluation, long term follow-up, and a randomized medical control group. Until further studies without these shortcomings are performed, the efficacy and safety of urgent EC-IC bypass for symptomatic ICA or MCA arteriosclerosis cannot be determined. 


\section{Reference List}

1. Hayashi T, Shirane R, Fujimura M, Tominaga T (2010) Postoperative neurological deterioration in pediatric moyamoya disease: watershed shift and hyperperfusion. $\mathrm{J}$ Neurosurg Pediatr 6:73-81.

2. Horiuchi T, Kusano Y, Asanuma M, Hongo K (2012) Posterior auricular artery-middle cerebral artery bypass for additional surgery of moyamoya disease. Acta Neurochir (Wien ) 154:455-456.

3. Horiuchi T, Nitta J, Ogiwara T, Sakai K, Hongo K (2009) Outcome predictors of open embolectomy in middle cerebral artery occlusion. Neurol Res 31:892-894.

4. Horiuchi T, Nitta J, Sakai K, Tanaka Y, Hongo K (2007) Emergency embolectomy for treatment of acute middle cerebral artery occlusion. J Neurosurg 106:257-262.

5. Hwang G, Oh CW, Bang JS, Jung CK, Kwon OK, Kim JE, Bae HJ, Han MK (2011) Superficial temporal artery to middle cerebral artery bypass in acute ischemic stroke and stroke in progress. Neurosurgery 68:723-729.

6. Murata T, Horiuchi T, Nitta J, Sakai K, Ogiwara T, Kobayashi S, Hongo K (2010) Urgent open embolectomy for cardioembolic cervical internal carotid artery occlusion. Neurosurg Rev 33:341-348.

7. Nussbaum ES, Janjua TM, Defillo A, Lowary JL, Nussbaum LA (2010) Emergency extracranial-intracranial bypass surgery for acute ischemic stroke. J Neurosurg 112:666-673

8. Powers WJ, Clarke WR, Grubb RL, Jr., Videen TO, Adams HP, Jr., Derdeyn CP (2011) Extracranial-intracranial bypass surgery for stroke prevention in hemodynamic cerebral ischemia: the Carotid Occlusion Surgery Study randomized trial. JAMA 306:1983-1992.

9. Rahmah NN, Horiuchi T, Kusano Y, Seguchi T, Hanaoka Y, Aoyama T, Hongo K Early changes of tissue perfusion after tissue plasminogen activator in hyperacute ischemic stroke: initial experiences with arterial spin labeling perfusion MRI. Neurol Med Chir (Tokyo) in press

10. Sakai K, Nitta J, Horiuchi T, Ogiwara T, Kobayashi S, Tanaka Y, Hongo K (2008) 
EC-IC bypass

Emergency revascularization for acute main-trunk occlusion in the anterior circulation. Neurosurg Rev 31:69-76.

11. Yoshimoto Y, Kwak S (1995) Superficial temporal artery--middle cerebral artery anastomosis for acute cerebral ischemia: the effect of small augmentation of blood flow. Acta Neurochir (Wien ) 137:128-137. 


\section{Figure legends}

Figure 1: Case 1. A-C: Diffusion-weighted images reveal small infarction area in the left hemisphere. D-E: Preoperative lateral view of left carotid angiograms showing middle cerebral artery occlusion and moderate collateral flow through anterior cerebral artery.

Figure 2: Case 1. Anteroposterior (A) and lateral (B) projections of left carotid artery injection showing a good blood supply through bypass.

Figure 3: Case 2. Preoperative magnetic resonance imaging (A) demonstrates no infarction at the basal ganglia. Anteroposterior (B), oblique (C), and lateral (C) projections of left carotid artery injection showing a severe stenosis (arrow) at the superior trunk and perforating arteries (arrowhead) originating from the stenotic lesion.

Figure 4: Case 2. Postoperative computed tomography (A and B) revealing newly developed infarction at the basal ganglia and coronal radiation, but not cortex. 

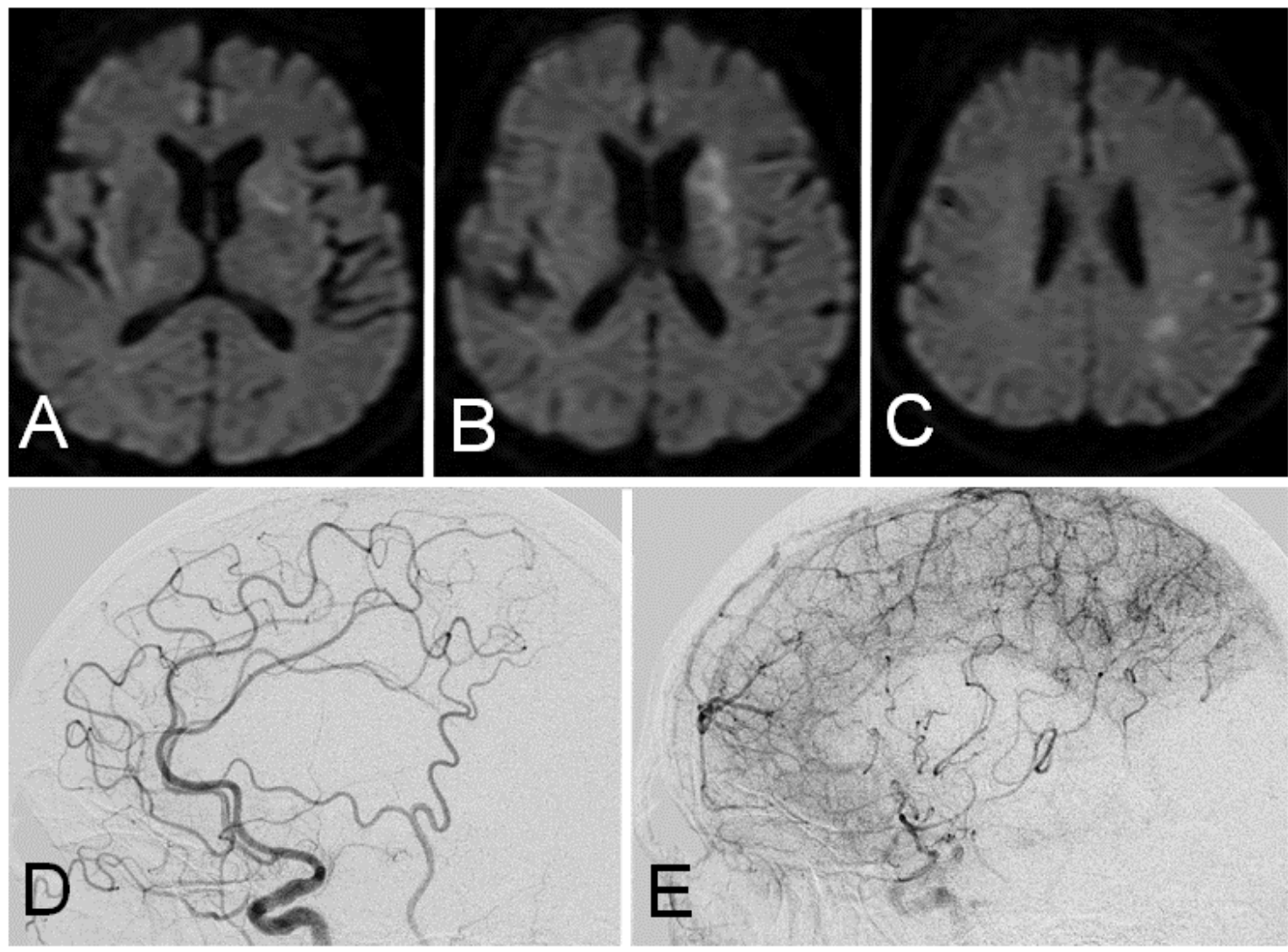

Figure 1
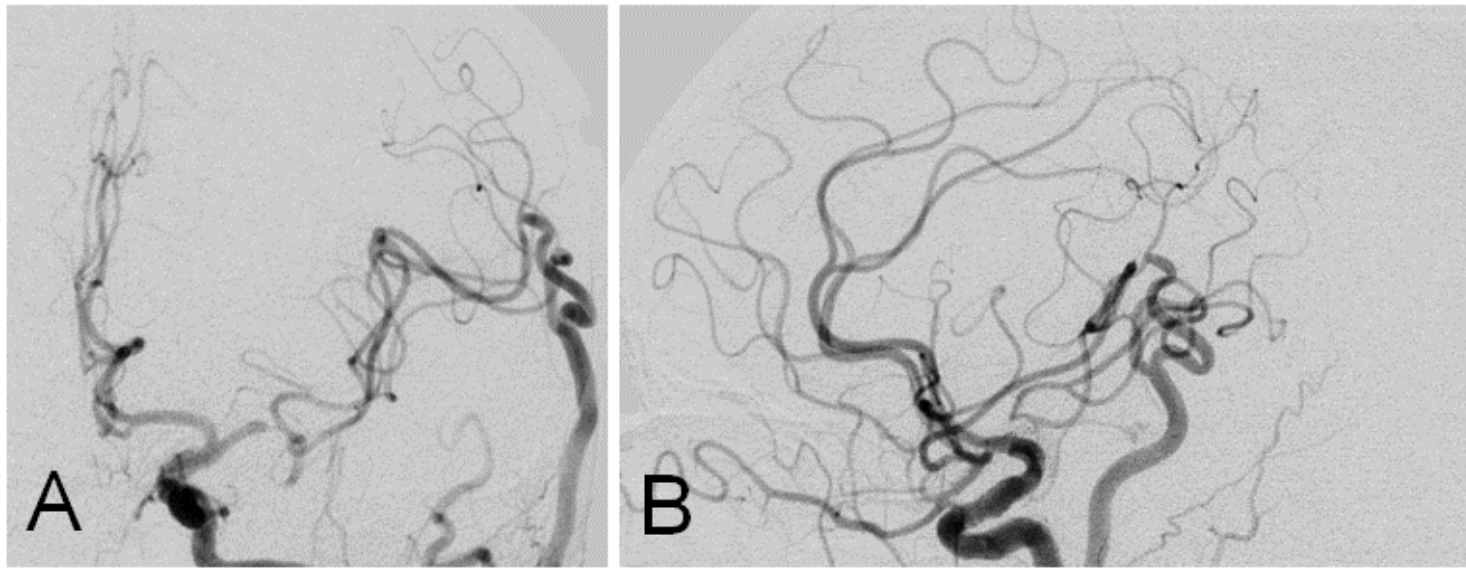

Figure 2 

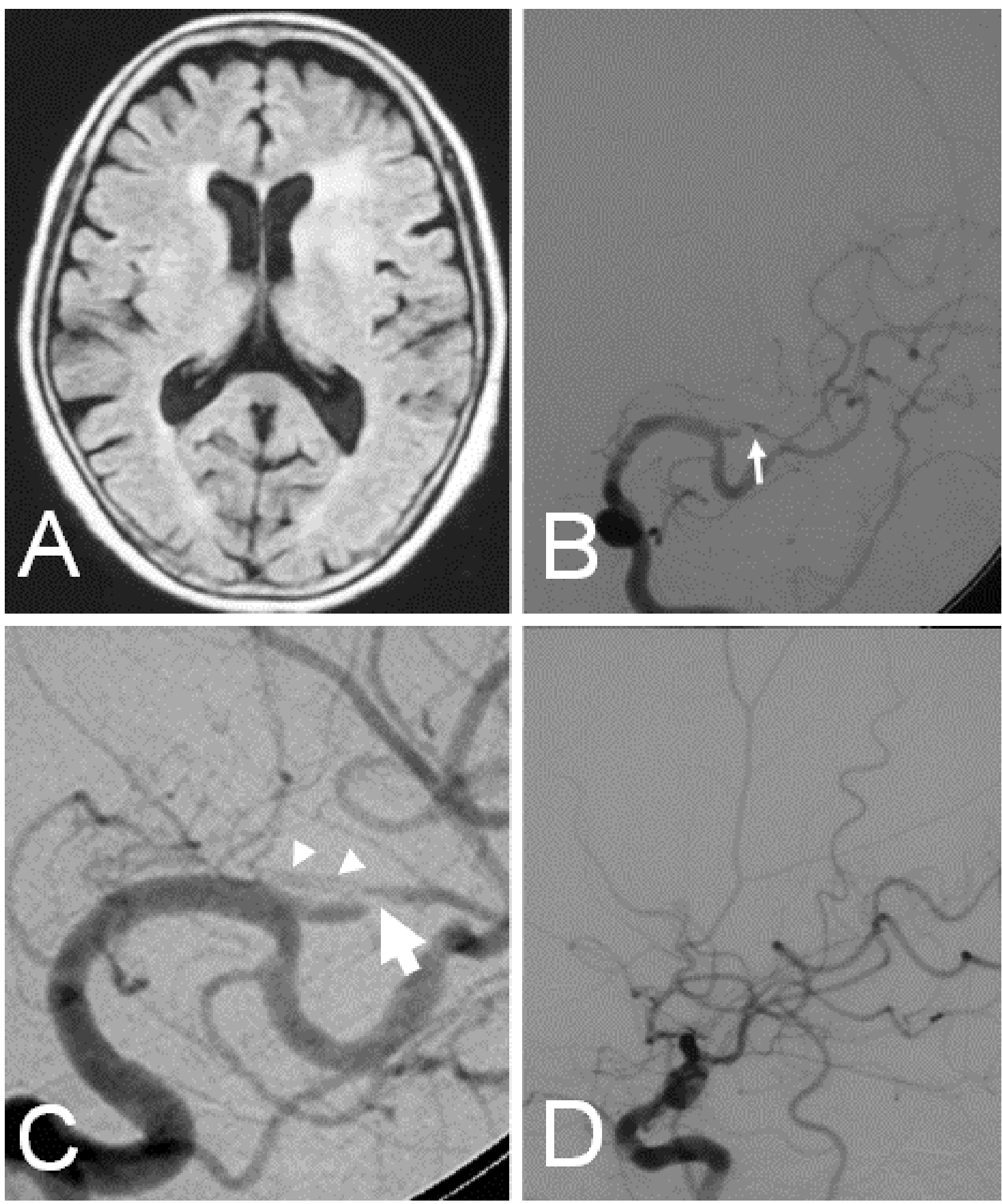

Figure 3 

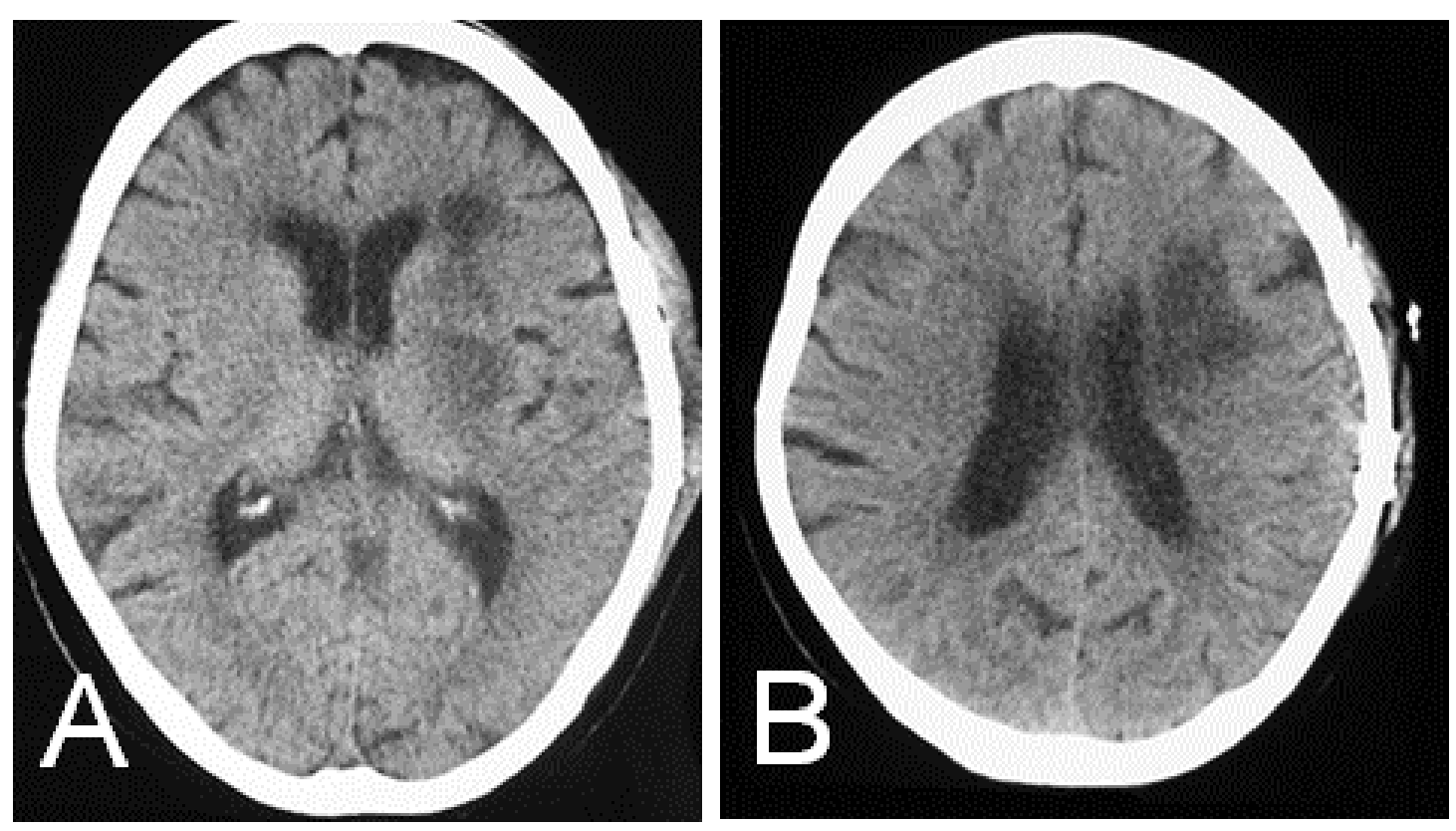

Figure 4 